OPEN ACCESS

Edited by:

Heiko Mühl,

Goethe University Frankfurt,

Germany

Reviewed by:

Kenichi Morikawa,

Hokkaido University Hospital,

Japan

Lynn B. Dustin,

University of Oxford,

United Kingdom

*Correspondence:

Alexander H. Dalpke

alexander.dalpke@med.uni-

heidelberg.de

Specialty section:

This article was submitted to Inflammation,

a section of the journal

Frontiers in Immunology

Received: 02 August 2017 Accepted: 19 September 2017 Published: 29 September 2017

Citation:

Klöss V, Grünvogel O, Wabnitz G, Eigenbrod T, Ehrhardt S,

Lasitschka F, Lohmann $V$ and Dalpke AH (2017) Interaction and

Mutual Activation of Different Innate Immune Cells Is Necessary to Kill and Clear Hepatitis C Virus-Infected Cells.

Front. Immunol. 8:1238. doi: 10.3389/fimmu.2017.01238

\section{Interaction and Mutual Activation of Different Innate Immune Cells Is Necessary to Kill and Clear Hepatitis C Virus-Infected Cells}

\author{
Volker Klöss' ${ }^{1}$ Oliver Grünvogel ${ }^{2}$, Guido Wabnitz ${ }^{3}$, Tatjana Eigenbrod' ${ }^{1}$, Stefanie Ehrhardt ${ }^{1}$, \\ Felix Lasitschka ${ }^{4}$, Volker Lohmann ${ }^{2}$ and Alexander H. Dalpke ${ }^{1 *}$ \\ 'Department of Infectious Diseases, Medical Microbiology and Hygiene, University Hospital Heidelberg, Heidelberg, \\ Germany, ${ }^{2}$ Department of Infectious Diseases, Molecular Virology, University of Heidelberg, Heidelberg, Germany, ${ }^{3}$ Section \\ Molecular Immunology, Institute of Immunology, University of Heidelberg, Heidelberg, Germany, ${ }^{4}$ Institute of Pathology, \\ University Hospital Heidelberg, Heidelberg, Germany
}

Innate immune cells can sense hepatitis $\mathrm{C}$ virus (HCV)-infected cells and respond with anti-viral actions including secretion of interferons (IFNs). In previous studies, the response of individual innate immune cells against HCV was analyzed in detail. We hypothesized that interaction of multiple innate immune cells increases the magnitude of the immune response and eventually leads to clearance of HCV-infected cells. To investigate this, we co-cultured Huh-7 HCV subgenomic replicon (SGR) cells with peripheral blood mononuclear cells (PBMCs). We confirm secretion of IFN $\alpha$ by plasmacytoid dendritic cells (pDCs) and IFN $\gamma$ by natural killer (NK) cells in the co-culture setup. Moreover, we observed that also monocytes contribute to the anti-viral response. Flow cytometry and ImageStream analysis demonstrated that monocytes take up material from HCV SGR cells in co-culture with PBMCs. Preceding the uptake, PBMCs caused apoptosis of HCV SGR cells by tumor necrosis factor-related apoptosis inducing ligand (TRAIL) expression on NK cells. We observed that only the interplay of monocytes, pDCs, and NK cells resulted in efficient clearance of HCV SGR cells, while these cell populations alone did not kill HCV SGR cells. Despite similar TRAIL receptor expression on Huh-7 control cells and HCV SGR cells, HCV activated PBMCs specifically killed HCV SGR cells and did not target Huh-7 control cells. Finally, we showed that HCV replicating cells per se are sensitive toward TRAIL-induced apoptosis. Our results highlight the importance of the interplay of different innate immune cells to initiate an efficient, rapid, and specific response against HCV-infected cells.

\footnotetext{
Keywords: hepatitis $\mathbf{C}$ virus, natural killer cell, monocyte, plasmacytoid dendritic cell, interferon, tumor necrosis factor-related apoptosis inducing ligand
}

\begin{abstract}
Abbreviations: HCV, hepatitis C virus; IFN, interferon; SGR, subgenomic replicon; PBMC, peripheral blood mononuclear cells; pDC, plasmacytoid dendritic cells; NK, natural killer cells; TRAIL, tumor necrosis factor-related apoptosis inducing ligand; LPS, lipopolysaccharide; JFH, Japanese fulminant hepatitis; DV, dengue virus; HAV, hepatitis A virus; G418, geneticin; CFSE, carboxyfluorescein succinimidyl ester; JC1, HCV chimera consisting of the strains J6CF and JFH-1; CD, cluster of differentiation; TNF, tumor necrosis factor; MOI, multiplicity of infection; MFI, mean fluorescence intensity; PARP, poly (ADP-ribose) polymerase; DR, death receptor; DcR, decoy receptor; TLR, toll-like receptor; IL, interleukin; LDH, lactate dehydrogenase.
\end{abstract}




\section{INTRODUCTION}

Hepatitis $\mathrm{C}$ virus (HCV) is a positive-strand RNA virus belonging to the family of Flaviviridae. Worldwide about 130-170 million people are infected by HCV and while around $25 \%$ of patients with acute infection spontaneously clear $\mathrm{HCV}, 75 \%$ develop chronic infection with risk for severe liver disease (1). The innate immune system, as the first line of defense, plays an important role for the immediate response against the invading pathogen. Indeed, several innate immune cells are present in or migrate to the liver upon HCV infection. Among others, plasmacytoid dendritic cells (pDCs) are enriched in HCV-infected livers (2). While in peripheral blood mononuclear cells (PBMCs) around $12 \%$ of the lymphocytes are natural killer $(\mathrm{NK})$ cells, in the liver, this number rises to $30 \%$ (3). Moreover, $15-25 \%$ of the cells in the liver are Kupffer cells, the resident liver macrophages $(4,5)$.

In recent years, activation of innate immune cells by HCV has drawn attention. As a model system Takahashi et al. co-cultured $\mathrm{HCV}$-infected cells with PBMCs and observed secretion of IFN $\alpha$ by pDCs (6). They recognized that this rapid activation could also be induced with HCV subgenomic replicon cells (HCV SGR) and that pDCs sense viral HCV RNA via TLR7. Later, it was shown that also monocytes and NK cells respond to HCV-replicating cells (7). Noteworthy, IFN $\gamma$ production by NK cells is dependent on monocytes (7) and on pDCs (8). Secretion of interferons (IFNs) in this co-culture is an important anti-viral mechanism, as IFNs stimulate the induction of interferon-stimulated genes, thereby inhibiting further viral replication (9-11). So far, these studies showed that multiple innate immune cells are activated by $\mathrm{HCV}$ and can limit viral replication. However, studies were limited to the analysis of the response of individual immune cell populations against HCV. Hence, most of the experiments were conducted with purified immune cells, yet interactions between innate immune cells will take place and probably are important for the overall activation state, as shown for NK cell activation by monocytes and pDCs $(7,8)$. We speculated that multiple interactions between different innate immune cells augment the overall activation state and thus exert a stronger anti-viral response.

In this study, we used co-culture systems of liver cell lines with acute and persistent HCV replication and PBMCs to investigate whether the interaction of multiple innate immune cells results in an efficient anti-viral response. While IFNs can limit HCV replication, we hypothesized that mutual interaction and activation between innate immune cells can lead to killing and clearance of HCV SGR cells. Since innate immune cells in the context of HCV infection are suspected to cause liver injury (12), we analyzed if $\mathrm{HCV}$ activated innate immune cells show specificity for targeting only HCV-infected cells.

\section{MATERIALS AND METHODS}

\section{Reagents, Inhibitors, and Blocking Antibodies}

R848 was purchased from InvivoGen (San Diego, CA, USA), lipopolysaccharide (LPS) from Salmonella minnesota was kindly provided by U. Seydel (Division of Biophysics, Research Center
Borstel, Borstel, Germany). Bafilomycin was from Calbiochem (Darmstadt, Germany). The pan-Caspase inhibitor Z-VAD-FMK was from InvivoGen, Caspase-8 inhibitor Z-IETD-FMK and Caspase-1 inhibitor Z-YVAD-FMK from Enzo Life Sciences (Lausen, Switzerland). TRAIL blocking antibody was from BD (Heidelberg, Germany, 550912) as well as the appropriate IgG control antibody (BD, 553447).

\section{Cells}

All Huh-7- and Huh-6-derived cell lines were cultured in Dulbecco's modified Eagle medium (DMEM) supplemented with $10 \%$ fetal bovine serum, $100 \mathrm{U} / \mathrm{ml}$ of penicillin, $100 \mathrm{ng} / \mathrm{ml}$ of streptomycin and non-essential amino acids (all from Thermo Fisher Scientific, Waltham, MA, USA). Cells were cultivated at $37^{\circ} \mathrm{C}$ and $5 \% \mathrm{CO}_{2}$. Naïve Huh-7 and Huh-7 9-13 cells harboring the HCV genotype $1 \mathrm{~b}$ replicon Con 1 were described previously (13) (GenBank accession no. AJ238799), the latter are designated Huh-7 Con 1 throughout this study. Cured Con 1 cells were generated by IFN $\alpha$ treatment of Huh-7 Con1 cells as described (14). Naïve Huh-6, Huh-6 JFH (HCV genotype 2a, AB047639) have been described (15), the latter were cured by treatment with direct acting antivirals (unpublished, A. Cerwenka, DKFZ, Heidelberg, Germany). Huh-7 cells with SGRs from dengue virus (Huh-7 DV, KU725663) (16) or from hepatitis A virus (Huh-7 HAV, M59808) (17) have been described before. Huh-7.5 cells were a kind gift by C. Rice (The Rockefeller University, New York, NY, USA) (18).

\section{PBMC Isolation}

Fresh human PBMCs were isolated from blood from voluntary healthy donors by standard Pancoll density-gradient centrifugation (PAN-Biotech GmbH, Aidenbach, Germany). PBMCs were directly cultured in RPMI 1640 (Biochrom, Berlin, Germany) supplemented with $10 \%$ fetal bovine serum. Overall blood from 30 different donors was used, yet individual experiments were done with 3-5 donors as indicated in the respective figure legend. Donors had no history of hepatitis. Blood sampling was approved by the ethics committee of the Medical Faculty Heidelberg and was done in accordance with their recommendations. All subjects gave written informed consent in accordance with the Declaration of Helsinki.

\section{Selection of SGR Cells}

To ensure constant selection for replicon cells, Huh-7 Con1 cells were cultured with $1 \mathrm{mg} / \mathrm{ml}$ of G418 (Carl Roth, Karlsruhe, Germany), Huh-6 JFH cells with $0.5 \mathrm{mg} / \mathrm{ml}$ of G418, Huh-7 DV cells with $75 \mu \mathrm{g} / \mathrm{ml}$ Hygromycin B (Thermo Fisher Scientific), and Huh-7 HAV cells with $2.5 \mu \mathrm{g} / \mathrm{ml}$ of Blasticidin S (Thermo Fisher Scientific).

\section{Co-Culture Setup}

For the co-culture of hepatoma cells with PBMCs, $1 \times 10^{5} \mathrm{Huh}$ cells were seeded in 48-well plates in DMEM. Upon adherence of Huh cells (after $4 \mathrm{~h}$ ), DMEM was removed and $1 \times 10^{6}$ PBMCs were added in RPMI. After overnight co-culture supernatant or cells were harvested for further analysis. When purified subsets of specific immune cells were used for co-culture with Huh cells, $1 \times 10^{4}$ pDCs, $1 \times 10^{5} \mathrm{NK}$ cells, or $1 \times 10^{5}$ monocytes were 
used. Accordingly, when these cells were depleted from PBMCs, those numbers were subtracted from $1 \times 10^{6}$ PBMCs. In triple co-culture experiments, $5 \times 10^{4} \mathrm{Huh}-7$ cells were seeded together with $5 \times 10^{4}$ Huh-7 Con 1 cells.

For flow cytometry analysis of the uptake of hepatoma cell remnants by monocytes, Huh-7 cells were stained with carboxyfluorescein succinimidyl ester (CFSE) (Sigma-Aldrich, Taufkirchen, Germany) or with Cytotell ${ }^{\mathrm{TM}}$ RED (CytoRED) (AAT Bioquest, Sunnyvale, CA, USA) prior to seeding. Cells were incubated with the intracellular dye CFSE ( $4.5 \mu \mathrm{M}$ in PBS) for $5 \mathrm{~min}$ at room temperature in the dark, washed once with PBS, once with DMEM and then were seeded. Using the same protocol hepatoma cells were also stained with Cytotell ${ }^{\mathrm{TM}} \mathrm{RED}$ (CytoRED) (AAT Bioquest, Sunnyvale, CA, USA) with a $0.5 \times$ dye working solution for triple co-culture experiments.

\section{Purification of Immune Cell Subsets}

Plasmacytoid dendritic cells, NK cells, and monocytes were purified or depleted from PBMCs by MACS separation technology with CD304, CD56, or CD14 specific magnetic beads, respectively (Miltenyi Biotec, Bergisch Gladbach, Germany).

\section{Cell Stimulation and HCV Infection}

Purified monocytes were co-cultured with Huh-7 cells in the presence of $700 \mathrm{pg} / \mathrm{ml}$ IFN $\alpha$ (Peprotech, Hamburg, Germany) or $7 \mathrm{ng} / \mathrm{ml} \mathrm{IFN} \gamma$ (Peprotech). To analyze sensitivity of Huh-7 cells for TRAIL and TNF $\alpha$ stimulation, Huh-7 cells were treated with 50 or $100 \mathrm{ng} / \mathrm{ml}$ of TRAIL (eBioscience) or TNF $\alpha$ (Peprotech) for the indicated time points (see figures).

For infection of Huh-7 cells with HCV, the JC1 virus was used as described (19). $5 \times 10^{4} \mathrm{Huh}-7$ cells were seeded in a 24 -well plate. $24 \mathrm{~h}$ later infection with a multiplicity of infection of 3 was performed for $6 \mathrm{~h}$. Cells were then washed and stained with CFSE as described above. After $72 \mathrm{~h}$, PBMCs were added to infected Huh-7 cells for overnight co-culture.

\section{Cytokine Secretion and Cytotoxicity}

Cytokine secretion was quantified by Sandwich-ELISA. Cell-free supernatants were harvested and IFN $\alpha$, IFN $\gamma$ (both eBioscience, Frankfurt, Germany), and interleukin (IL)-6 (BD, Heidelberg, Germany) levels were measured. Cell-free supernatants were also used to measure immune cell mediated cytotoxicity of Huh-7 cells by detection of lactate dehydrogenase $(\mathrm{LDH})$ release with the Cytotoxicity Detection Kit ${ }^{\text {Plus }}$ (Roche, Mannheim, Germany).

\section{Flow Cytometry Antibodies}

Analysis of CD80 or CD86 expression on monocytes was done with CD14-APC (BD, 555399), CD80-PE (BD, 557227), or CD86-PE (BD, 555665). For detection of CD25, CD69, and TRAIL on NK cells, NK cells were identified as $\mathrm{CD} 6^{+} / \mathrm{CD}^{-}$. The following stainings were performed: CD25-PerCP-Cy5.5 (Biolegend, San Diego, CA, USA, 356112), CD56-APC (Miltenyi Biotec, 130-090843), and CD3-FITC (BD, 555332). CD69-APC (BD, 555533), CD56-Pe-Cy7 (eBioscience, 25-0567-42), and CD3-FITC (BD, 555332). TRAIL-PE (BD, 550516), CD56-APC (Miltenyi Biotec, 130-090-843), and CD3-APC-Cy7 (BD, 557832). Samples were measured on a BD FACSCanto II with BD FACSDIVA 8 software.

\section{Classical and Imaging Flow Cytometry Analysis}

To analyze the uptake of CFSE positive Huh cells by monocytes, monocytes were stained with CD14-PE (BD, Heidelberg, Germany, 562691) in FACS buffer (2\% FCS in PBS) and uptake was quantified by classical flow cytometry. Samples were measured on a BD FACSCanto II with BD FACSDIVA 8 software.

Imaging flow cytometry (hereafter termed ImageStream) was performed as described (20). Cells were analyzed by ImageStream (Amnis, Merck Millipore, Darmstadt, Germany). Further antibodies used for flow cytometry are described in Data Sheet S1 in Supplementary Material.

\section{Western Blot Analysis}

To analyze induction of apoptosis in Huh-7 cells cleaved poly (ADP-ribose) polymerase (PARP) was detected by Western blot. After co-culture with PBMCs or incubation with recombinant TRAIL, Huh-7 cells were washed once with PBS, lysed in Laemmli buffer, and incubated at $95^{\circ} \mathrm{C}$ for $10 \mathrm{~min}$. Proteins were separated by SDS-PAGE and transferred to a nitrocellulose membrane by semidry blotting. Unspecific binding was blocked by incubating the membranes in 5\% dry milk in 1xTBST (TBS, 0.05\% Tween-20) for $1 \mathrm{~h}$. Primary antibodies against cleaved PARP (Cell Signaling, Leiden, Netherlands, \#9541) and $\beta$-actin (Cell Signaling, \#4970) were diluted in blocking buffer 1:1,000 and 1:5,000, respectively. Membranes were incubated with primary antibody overnight at $4^{\circ} \mathrm{C}$, washed three times for $10 \mathrm{~min}$ with $1 \times \mathrm{TBST}$ at RT, and incubated with HRP-conjugated anti rabbit antibody (Cell Signaling, \#7074) diluted 1:4,000 in blocking buffer for $45 \mathrm{~min}$ at RT. Again, membranes were washed as described above and proteins were then detected by using enhanced chemiluminescence (ECL) substrate (PerkinElmer, Rodgau, Germany). Densitometric analysis was performed using ImageJ software (National Institutes of Health, Bethesda, MD, USA). Original image data are shown as attachment in the Data Sheet S1 in Supplementary Material.

\section{Quantitative RT-PCR}

Cellular RNA was extracted with the peqGold Total RNA Kit (peqlab Biotechnology, Erlangen, Germany). RNA was transcribed into cDNA using High Capacity cDNA RT Kit (Applied Biosystems, Foster City, CA, USA). RT-PCR was set up with $\mathrm{SYBR}^{\circledR}$ Green PCR Master Mix Fast (Applied Biosystems) and specific primers for each target (DR4 forward: 5' AGAGAGAAGTCCCTGCACCA 3', DR4 reverse: 5' GTCACTCCAGGGCGTACAAT 3', DR5 forward: 5' AAGACCCTTGTGCTCGTTGT 3', DR5 reverse: 5' AGGTGGACACAATCCCTCTG 3', DcR2 forward: 5' TACC ACGACCAGAGACACC 3', DcR2 reverse: 5' CACCCTGTTC TACACGTCCG $3^{\prime}, \beta$-actin forward: $5^{\prime}$ GGCTCCGATATCT CTGTCGT3', $\beta$-actin reverse: 5' ATGTTGCATTTCGTCACACC $\left.3^{\prime}\right)$. Analysis was performed on a StepOne Plus RT-PCR cycler (Applied Biosystems). Specificity of PCR was controlled by notemplate and no-RT control samples and by melting curves. The relative expression of each target gene as compared to $\beta$-actin was calculated. 


\section{Statistics}

Experiments were repeated as indicated in the figure legends. Data are shown as mean $+\mathrm{SD}$. Statistical significance was analyzed by ANOVA using R 3.0.2 (R Foundation for Statistical Computing, Vienna, Austria) followed by a two-sided Tukey post hoc test. Significant differences were considered at $\mathrm{p}$ values of less than $\left.0.05{ }^{*}\right), 0.01\left(^{* *}\right), 0.001\left(^{* *}\right)$, no significance is indicated by ns.

\section{RESULTS}

\section{Plasmacytoid DCs and NK Cells Secrete IFNs in Response to HCV SGR Cells}

Hepatitis C virus SGR cells (HCV SGR) have been reported to stimulate PBMCs in a process involving transfer of viral RNA from HCV SGR cells into PBMCs (6). To study the activation in more detail, we set up a co-culture system of HCV SGR cells and complete human PBMCs. In order to avoid potential transmission of viral RNA by viral particles, SGR cells were preferred over $\mathrm{HCV}$-infected cells. We observed secretion of IFN $\alpha$ in co-cultures of the HCV SGR cell line Huh-7 Con 1 with PBMCs (Figure 1A, left). IFN $\alpha$ was neither detected in co-cultures of Huh-7 control cells with PBMCs, nor when Huh-7 Con 1 cells that were cured from the replicon ("cured Con1") were used. Similar to the production of IFN $\alpha$, IFN $\gamma$ was only secreted in co-cultures of Huh-7 Con 1 cells and PBMCs (Figure 1B, left).

To identify the cellular source of IFN $\alpha$ and IFN $\gamma$, distinct cell populations were depleted from PBMCs before stimulating with Huh-7 Con 1 cells. Upon depletion of pDCs, IFN $\alpha$ was not secreted anymore in co-culture supernatants (Figure 1A, right). IFN $\gamma$ production was abolished when NK cells were depleted from PBMCs (Figure 1B, right). The results show that $\mathrm{pDCs}$ and NK cells are activated by HCV SGR cells and respond by secreting IFN $\alpha$ and IFN $\gamma$, respectively.

We then investigated the mode of activation of PBMCs by HCV SGR cells. Earlier reports $(6,21)$ suggested a role for TLR7 recognizing viral RNA. Indeed, blocking nucleic acid recognizing endosomal TLRs using Bafilomycin resulted in complete loss of IFN $\alpha$ and IFN $\gamma$ production (Figures 1C,D). Bafilomycin did not reduce IL-6 secretion by PBMCs treated with LPS (Figure S1 in Supplementary Material), triggering non-endosomal TLR4. Mechanistically, it was suggested that cell-cell contacts but also exosomes are required to initiate the innate immune response $(6,22)$. We observed no trans-activation when Huh-7 Con 1 cells were separated from PBMCs by a Transwell insert nor could we stimulate PBMCs with concentrated supernatants of replicon cells (data not shown). Moreover, the production of IFN $\alpha$ and IFNy was significantly reduced when tight cell-cell contacts were inhibited by continuous mechanical shaking (Figures 1E,F). These findings support the hypothesis that innate immune cells are activated by viral RNA derived from infected hepatocytes in a manner that depends on close cell-cell contacts.

\section{Monocytes Take Up Material from HCV SGR Cells}

During the characterization of cytokine responses of PBMCs with HCV SGR cells, we consistently observed by microscopy that the
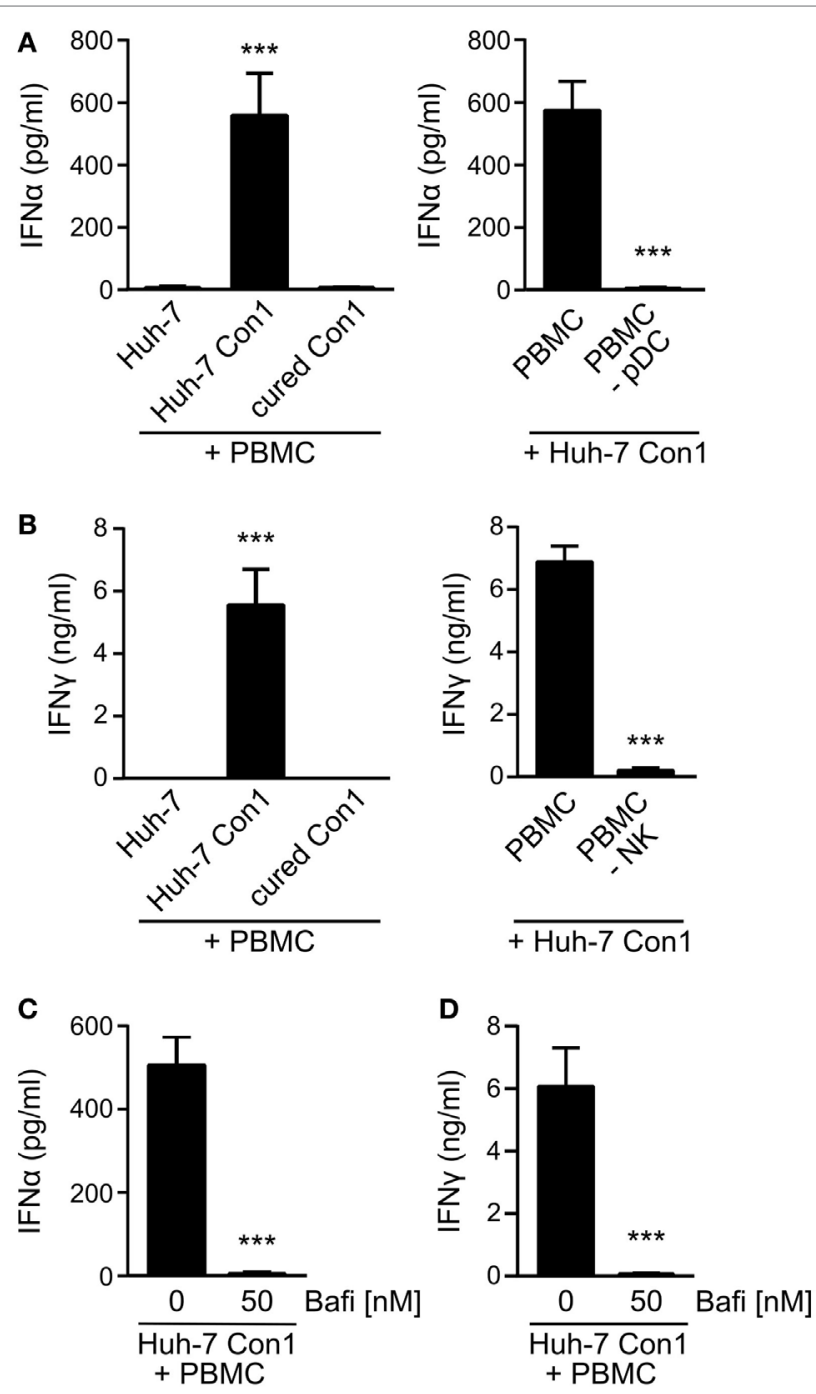

E

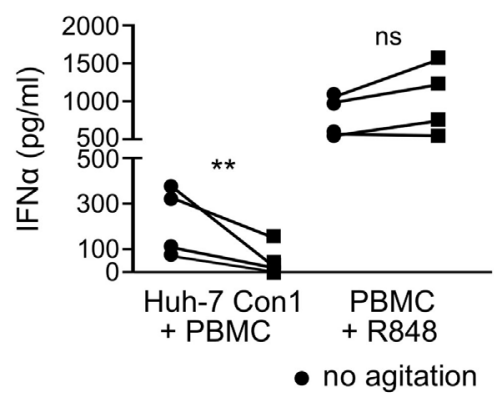

$\mathbf{F}$

FIGURE 1 | Plasmacytoid dendritic cells (pDCs) and natural killer (NK) cells are activated by hepatitis $C$ virus subgenomic replicon cells. Huh-7 cells were co-cultured with peripheral blood mononuclear cells (PBMCs) overnight and cytokines measured by ELISA. (A) IFN $\alpha$ levels in co-cultures with PBMCs or with PBMCs depleted from pDCs (PBMC -pDC) $(n=3)$. (B) IFN $\gamma$ levels in co-cultures with PBMCs or with PBMC depleted from NK cells (PBMC -NK) $(n=3)$. (C,D) IFN $\alpha$ and IFN $\gamma$ secretion in PBMCs that were treated with Bafilomycin (Bafi) for $1 \mathrm{~h}$ before setting up co-cultures $(n=3)$. (E,F) IFN $\alpha$ and IFN $\gamma$ levels in co-cultures that underwent continuous mechanical agitation (orbital shaker, $200 \mathrm{rpm}$ ) compared to co-cultures without agitation. PBMCs stimulated with R848 $(1 \mu \mathrm{g} / \mathrm{ml})$ were used as control. 
number of Huh-7 Con 1 cells declined during the co-culture period (data not shown). Further investigations of this decline revealed a novel contribution of monocytes to the immune response against HCV SGR cells. When Huh-7 Con1 cells were stained with CFSE prior to addition of PBMCs, monocytes (identified by expression of CD14) became CFSE positive after overnight co-culture (Figure 2A, right plot). In contrast, monocytes were CFSE negative when PBMCs were co-cultured with CFSE labeled Huh-7 cells that lack the SGR (Figure 2A, left plot). To analyze this in detail, we performed ImageStream analysis. Co-culture of CFSE stained Huh-7 cells and PBMCs resulted in few doublepositive cells (Figure 2A), which could be identified mostly as cell pairs (Figure 2B, upper row). In contrast, in the co-culture of Huh-7 Con 1 cells with PBMCs, we observed that most of the cells double-positive for CD14 and CFSE consisted of monocytes that had taken up particles from Huh-7 Con 1 cells (Figure 2B, lower row; Figure S2 in Supplementary Material). The size of these particles ranged from $1.8-3.3 \mu \mathrm{m}$ in diameter, suggesting that activated PBMCs induced apoptosis of HCV SGR cells. Induction of apoptosis was supported by the observation that the number of Huh-7 Con 1 cells significantly dropped compared to the number of Huh-7 cells in co-culture with PBMCs (shown in Figure 2A Q4, quantified in Figure 2C).
In order to quantify the uptake of CFSE stained Huh-7 cells by monocytes, the percentage of CFSE positive monocytes as well as the CFSE mean fluorescence intensity (MFI) of monocytes were analyzed. In co-cultures of Huh-7 Con 1 cells with PBMCs $83 \%$ of the monocytes became CFSE positive, whereas in control cells this percentage was $14 \%$ with Huh- 7 and $26 \%$ with cured Con 1 cells (Figure 3A). To confirm these findings, another cell line containing a replicon of another HCV genotype (Huh-6 $\mathrm{JFH}$, genotype 2a) was used with similar results (Figure 3B). As a proof-of-principle uptake experiments were also performed with HCV-infected cells instead of HCV SGR cells. Strikingly, the same phenotype was observed with HCV JC1-infected Huh-7.5 cells (Figure 3C). We next analyzed whether this uptake could also be observed with other RNA viruses. However, for dengue virus and HAV SGR cells, no significant uptake was observed (Figure 3D). Similar interpretations could be drawn when analyzing the MFI data (Figure S3 in Supplementary Material). We speculated that DV or HAV SGR cells secrete less viral RNA, thus explaining the lack of immune cell activation. Intracellular viral RNA levels were comparable in HCV, DV, and HAV SGR cells (Figure 3E). However, in contrast to HCV SGR cells, secretion of viral RNA by DV and HAV SGR cells indeed was significantly lower (Figure 3F). Of note, the uptake by monocytes was only observed with HCV

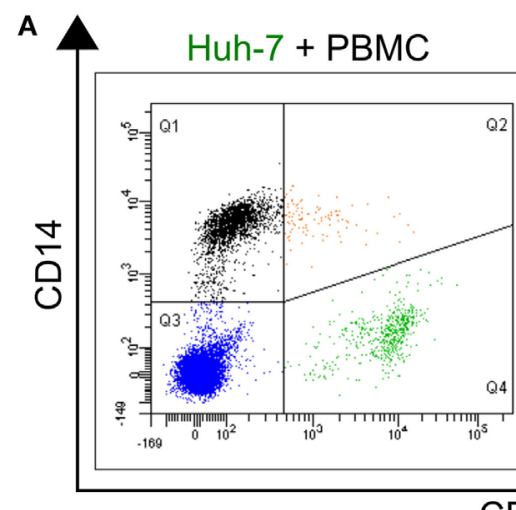

\section{Huh-7 Con1 + PBMC}

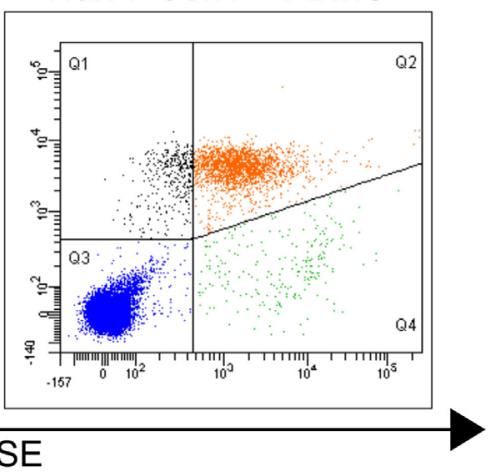

B

Nuclei

CD14

CFSE

Merge
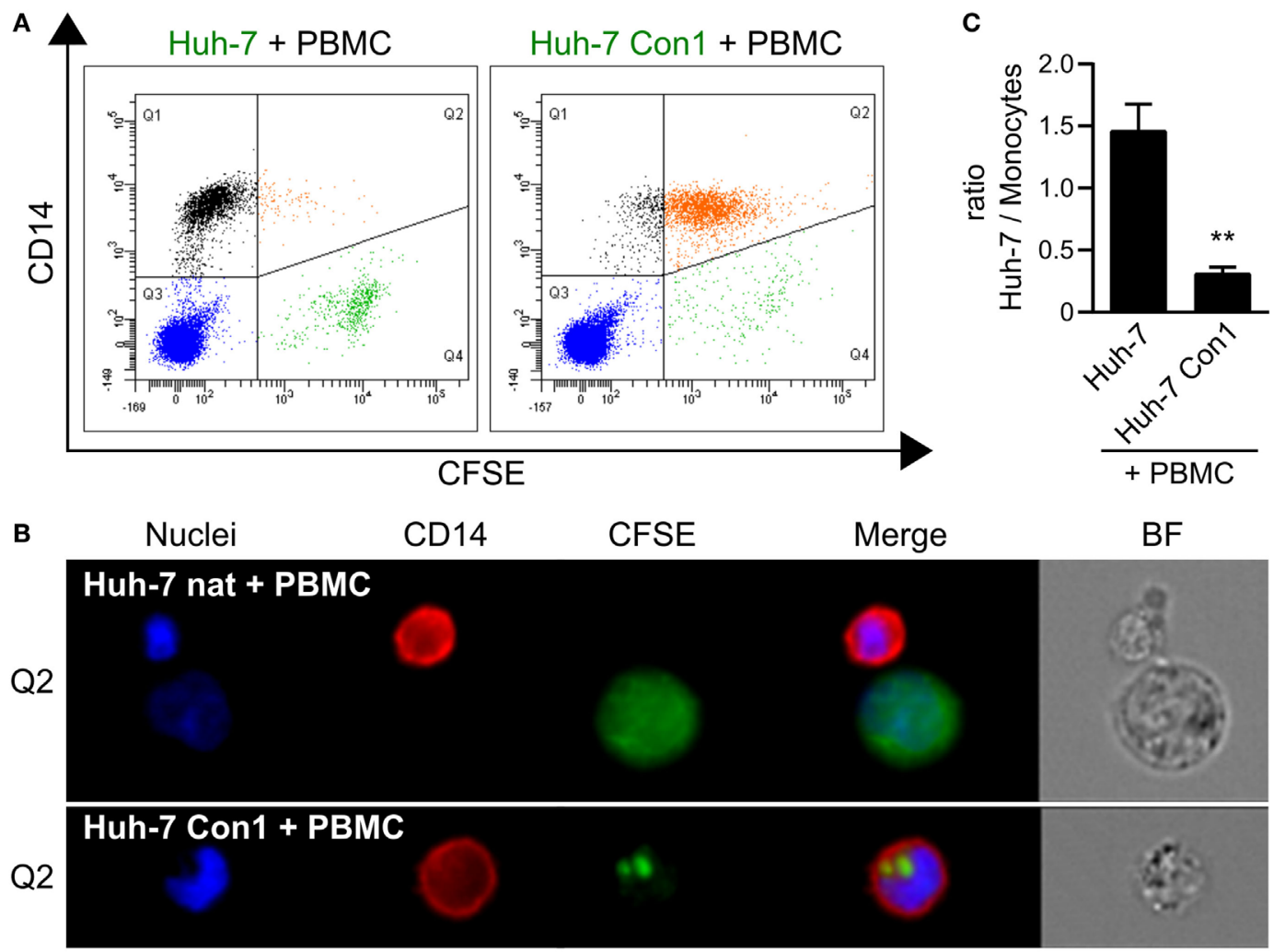

FIGURE 2 | Phagocytosis of particles from hepatitis C virus subgenomic replicon cells by monocytes. Carboxyfluorescein succinimidyl ester (CFSE) stained Huh-7 cells were co-cultured with peripheral blood mononuclear cells (PBMCs). (A) Analysis of CFSE distribution in relation to monocytes (CD14+). (B) Uptake of Huh-7 cells by monocytes depicted by ImageStream analysis. (C) Ratio between Huh-7 cells and monocytes after co-culture based on flow cytometry analysis in (A) $(n=3)$. 


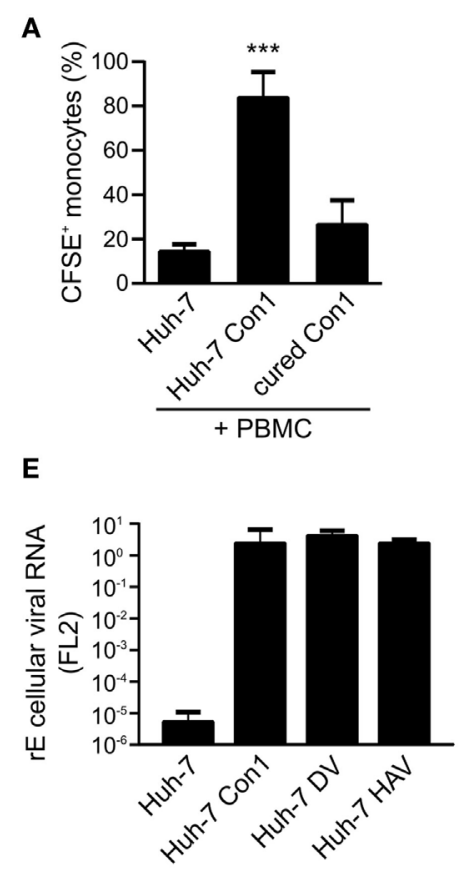

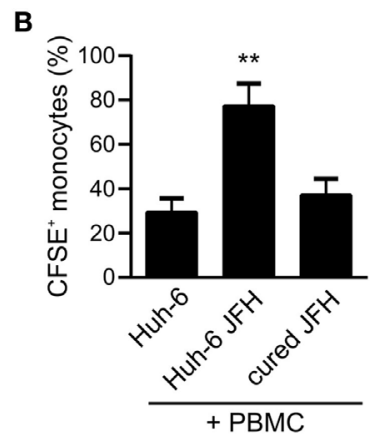

$\mathbf{F}$

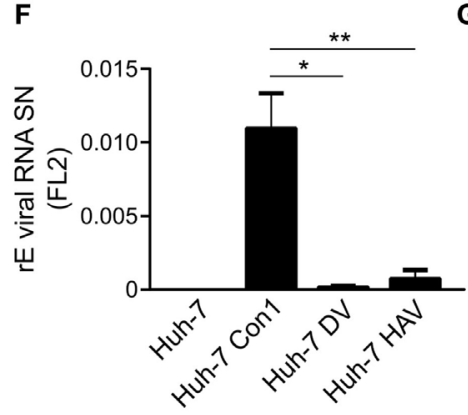

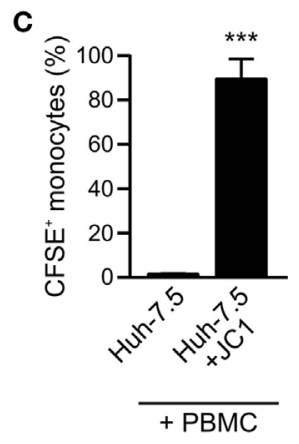

G

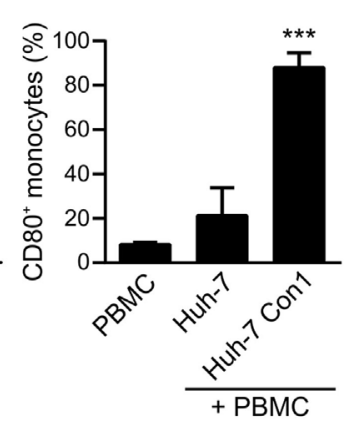

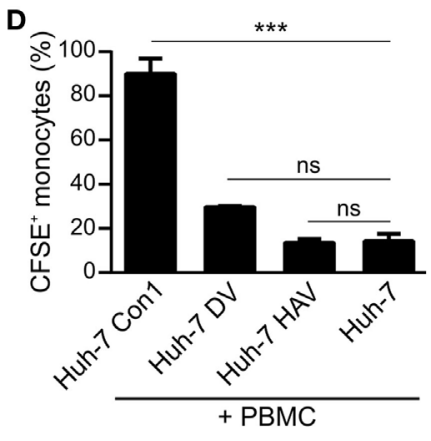

H

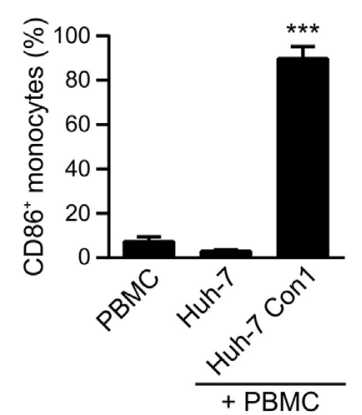

FIGURE 3 | Phagocytosis of hepatitis C virus subgenomic replicon particles by monocytes is specific for HCV. (A-D) Peripheral blood mononuclear cells (PBMCs) were co-cultured with various carboxyfluorescein succinimidyl ester (CFSE)-stained Huh cells and the percentage of CFSE ${ }^{+}$monocytes was determined by flow cytometry. (A) Co-cultures of PBMCs with Huh-7 cells (Con1 = HCV genotype 1b) $(n=3)$. (B) Co-cultures of PBMCs with Huh-6 cells (JFH = HCV genotype 2a) $(n=3)$. (C) Co-cultures of PBMCs with Huh-7.5 cells or with HCV-infected Huh-7.5 cells (Huh-7.5 + JC1) ( $n=2)$. (D) Co-cultures of PBMCs with Huh-7 cells, Huh-7 Con1 (HCV), Huh-7 DV (dengue virus), or Huh-7 hepatitis A virus (HAV) SGR cells $(n=3)$. (E,F) Huh-7 and Huh-7 SGR cells (Con1, DV, and HAV) were seeded for quantification of intracellular viral RNA (E) and secreted viral RNA in the supernatant (F) was measured by qPCR. RNA lysis buffer was spiked with Firefly Luciferase 2 (FL2) in vitro transcript for normalization $(n=3)$ ). $(\mathbf{G}, \mathbf{H})$ Huh-7 cells were co-cultured with PBMCs overnight and monocytes were analyzed for activation by expression of CD80 and CD86 by flow cytometry $(n=4)$.

SGR cells. Furthermore, we observed increased expression of the monocyte activation markers CD80 and CD86 in co-cultivation of Huh-7 Con 1 cells with PBMCs (Figures 3G,H). Our data show that next to pDCs and NK cells also monocytes are activated by HCV SGR cells and contribute to the anti-viral response.

\section{Uptake of HCV SGR Cells Requires Presence of pDCs and NK Cells}

As monocytes within complete PBMCs were taking up Huh-7 Con 1 cells, we addressed the question whether this phenotype was induced in a monocyte-intrinsic manner or whether it required additional cells. In co-cultures of purified monocytes with Huh-7 Con 1 cells, $25 \%$ of monocytes became CFSE positive (6\% with Huh-7, Figure 4A) and showed slightly increased CFSE MFI (Figure 4B). However, compared to monocytes within full PBMCs, the efficacy of uptake was clearly reduced with purified monocytes. Hence, we speculated that other innate immune cells contribute. As pDCs and NK cells were activated and secreted their respective IFNs in response to HCV SGR cells, we depleted those cells from PBMCs and analyzed the uptake of HCV SGR cell remnants by monocytes. Surprisingly, pDC or NK depletion alone did not reduce uptake. Only when both, pDCs and NK cells, were depleted the uptake by monocytes was significantly decreased (Figures 4C,D). Addition of recombinant IFN $\alpha$ or IFN $\gamma$ (to mimic functional activities of either pDCs or NK cells) to the co-culture of purified monocytes with Huh-7 Con 1 cells significantly increased the monocyte uptake (Figures 4E,F). Yet no synergistic effect was observed when combining both cytokines (data not shown). Neither IFN $\alpha$ nor IFN $\gamma$ induced an uptake of Huh-7 control cells by monocytes, indicating that IFNs did not unspecifically kill Huh-7 cells. We show that monocytes by themselves respond to HCV SGR cells, but the uptake by monocytes is dramatically stronger in presence of pDCs and NK cells and can be mimicked by addition of IFNs.

\section{PBMCs Kill HCV SGR Cells}

ImageStream analysis showed that monocytes take up small particles of Huh-7 Con 1 cells (Figure 2B). We speculated that those particles were remnants of Huh-7 Con1 cells that were killed by PBMCs. To support this hypothesis, an LDH release assay was performed. $36 \%$ of Huh-7 Con 1 cells were killed in co-culture with PBMCs, while no $\mathrm{LDH}$ was released upon co-culture of PBMCs with Huh-7 control cells (Figure 5A). To analyze if Huh-7 Con1 cells undergo apoptosis in co-culture with PBMCs, PARP cleavage determining the final irreversible step of apoptosis was analyzed. Cleaved PARP was only detected in co-culture of PBMCs with Huh-7 Con1, but not with Huh-7 

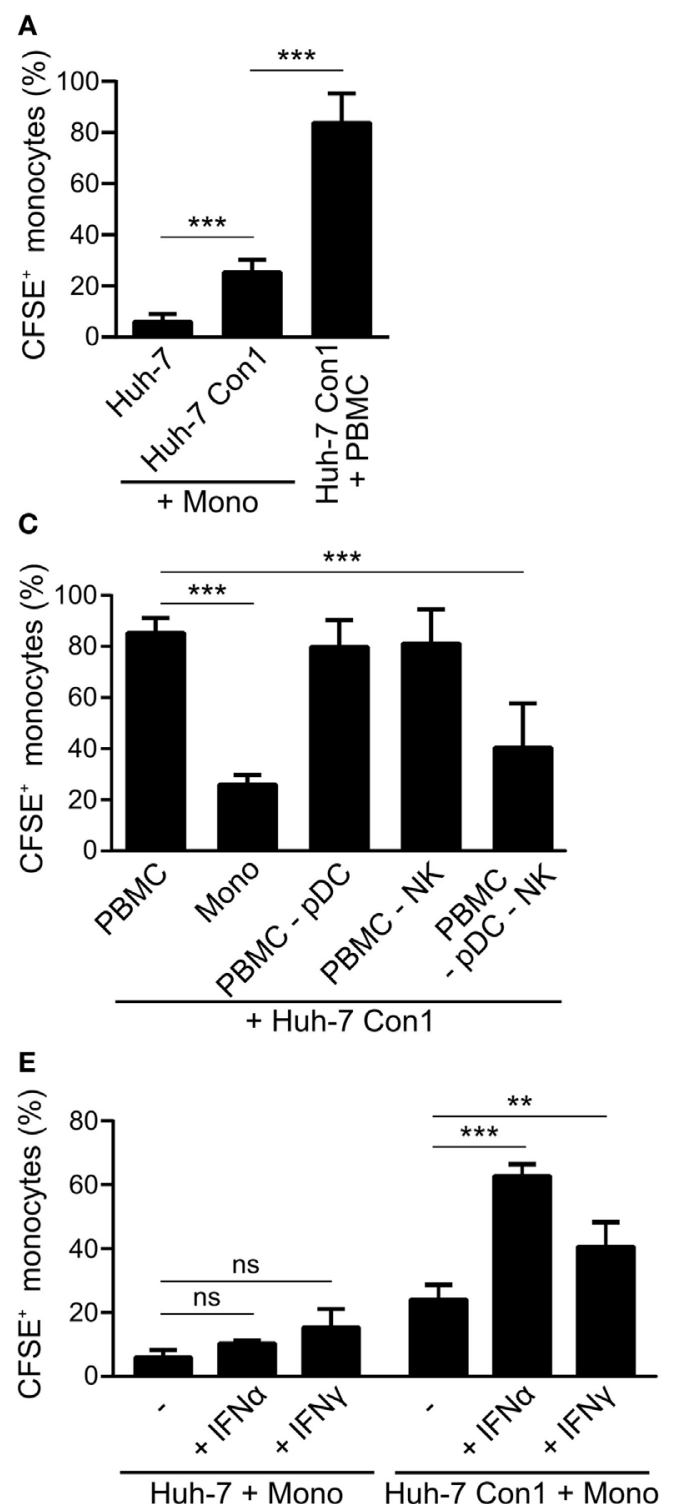
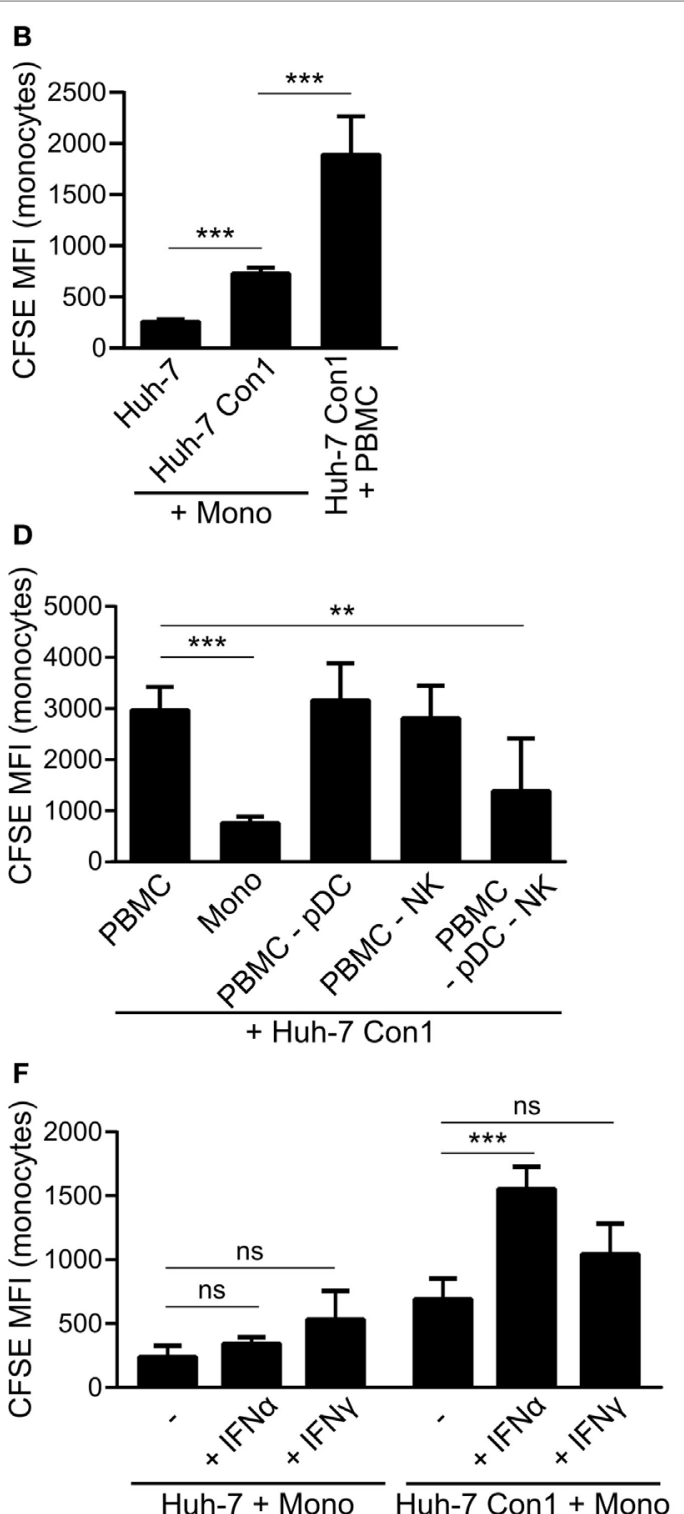

FIGURE 4 | Plasmacytoid dendritic cells (pDCs) and natural killer (NK) cells promote phagocytosis of hepatitis C virus subgenomic replicon by monocytes. (A,B) Purified monocytes (Mono) or peripheral blood mononuclear cells (PBMCs) were co-cultured with carboxyfluorescein succinimidyl ester (CFSE) stained Huh-7 cells overnight. Percentage of CFSE ${ }^{+}$monocytes $\mathbf{( A )}$ and CFSE mean fluorescence intensity (MFI) of monocytes $(\mathbf{B})$ was determined $(n=5)$. $(\mathbf{C}, \mathbf{D})$ CFSE stained Huh-7 Con1 cells were co-cultured overnight with PBMCs, purified monocytes (Mono), PBMCs depleted from pDCs (PBMC -pDC), PBMCs depleted from NK cells (PBMC -NK) or with PBMC depleted from pDCs and NK cells (PBMC -pDC -NK). CFSE+ monocytes were determined ( $n=3$ ). (E,F) Purified monocytes were co-cultured with CFSE stained Huh-7 cells in presence of recombinant IFN $\alpha(700 \mathrm{pg} / \mathrm{ml})$ or recombinant IFN $\gamma\left(7 \mathrm{ng} / \mathrm{ml}^{2}\right.$. CFSE ${ }^{+} \mathrm{monocytes}$ were determined $(n=4)$.

cells (Figure 5B). Since apoptosis in target cells is mediated by successive caspase activation, we tested if caspase inhibition protects Huh-7 Con 1 cells from apoptosis. Inhibition of all caspases (pan-Caspase inhibitor) decreased killing of Huh-7 Con1 cells from 29 to $4.6 \%$ (Figure 5C). Blocking Caspase-8, which is the first caspase activated upon induction of apoptosis, reduced killing to $18 \%$. Caspase-1, which is not involved in apoptosis but mediates inflammasome activation, had no effect on PBMC mediated killing of Huh-7 Con 1 cells. In consequence, blocking of apoptosis reduced the uptake of CFSE labeled Huh-7 SGR cells by monocytes (Figures 5D,E), proving that killing of Huh-7 Con 1 cells precedes the uptake by monocytes.

\section{Innate Immune Cells Interact to Mediate Killing of HCV SGR Cells}

As we observed that PBMCs killed HCV SGR cells, we analyzed which cells within PBMCs cause apoptosis in Huh-7 Con 1 cells. NK cells, pDCs or monocytes alone were not able to kill Huh-7 Con 1 cells, only recombination of purified pDCs, NK cells, and 


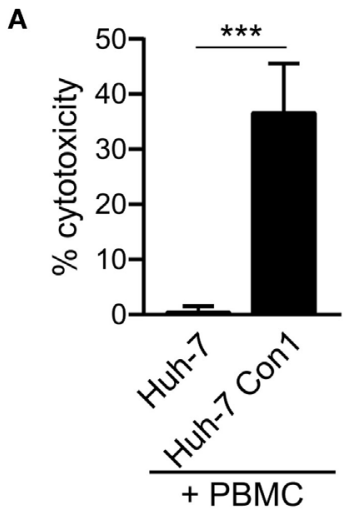

B

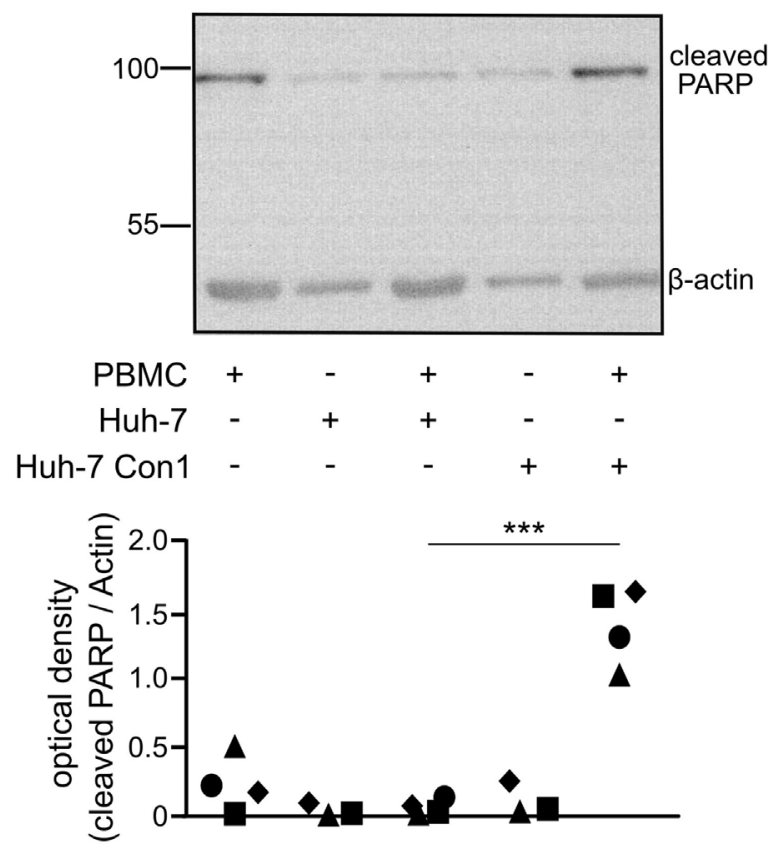

C

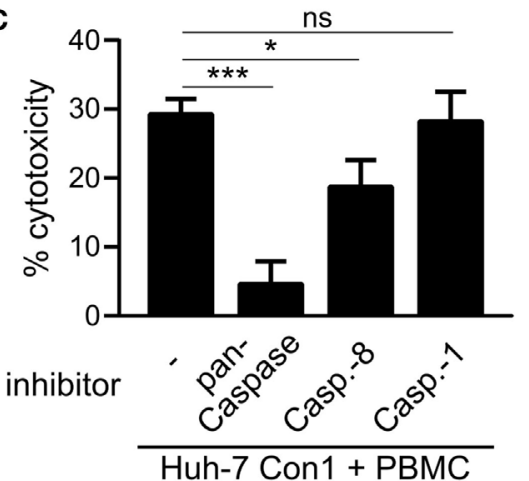

D

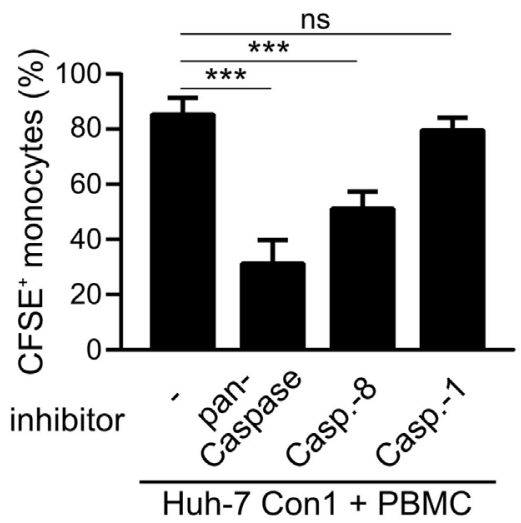

E

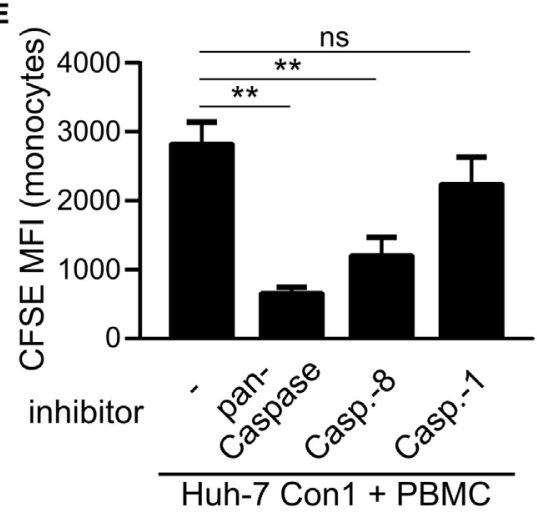

FIGURE $\mathbf{5}$ | Killing of hepatitis C virus subgenomic replicon cells by peripheral blood mononuclear cells (PBMCs). Huh-7 cells were co-cultured with PBMCs overnight. (A) Supernatants were collected and measured for lactate dehydrogenase $(\mathrm{LDH})$ activity $(n=5)$. (B) Cells were harvested and poly (ADP-ribose) polymerase (PARP) cleavage was analyzed by Western Blot $(n=3-4)$. (C-E) Huh-7 Con1 cells were co-cultured with PBMCs in presence of pan-Caspase inhibitor Z-VAD-FMK $(20 \mu M)$, Caspase-8 inhibitor Z-IETD-FMK $(20 \mu M)$, or Caspase-1 inhibitor Z-YVAD-FMK $(20 \mu M)$. After overnight incubation LDH release was measured in the supernatant (C), percentage of $\mathrm{CFSE}^{+}$monocytes (D) and carboxyfluorescein succinimidyl ester (CFSE) mean fluorescence intensity (MFI) of monocytes (E) was determined by flow cytometry $(n=4-5)$.

monocytes resulted in killing of Huh-7 Con 1 cells (Figure 6A). However, killing was not as efficient as with complete PBMCs, which might be due to functional impairment when purifying single cells. Next, pDCs, NK cells, or monocytes were depleted from PBMCs before setting up the co-culture with Huh-7 Con 1 cells. $\mathrm{LDH}$ release assays revealed that each cell subset contributed to killing, but none was solely responsible (Figure 6B). Only when both pDCs and NK cells were depleted, killing was significantly reduced. The data confer that interaction of multiple innate immune cells is necessary for an efficient anti-viral response.

\section{NK Cells Drive Apoptosis in HCV SGR Cells via TRAIL Expression}

Analyzing the contribution of the different cell subsets in more detail we found an upregulation of the NK cell activation markers 


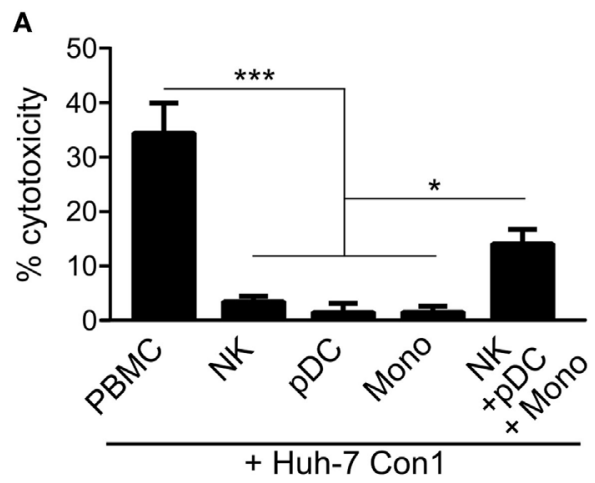

C

CD69

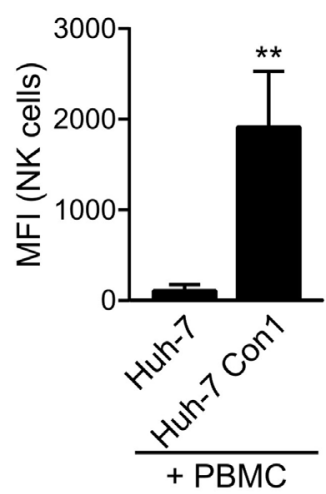

D

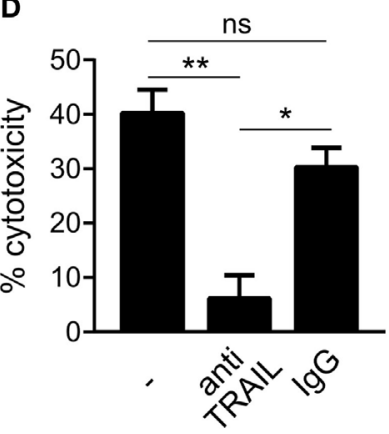

$\overline{\text { Huh-7 Con1 + PBMC }}$

E

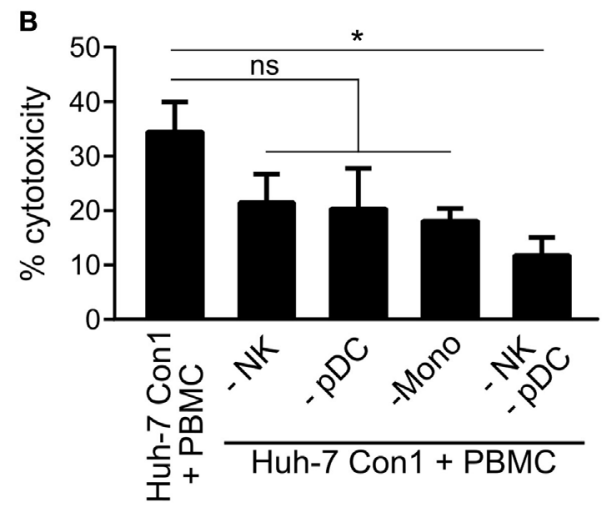

CD25

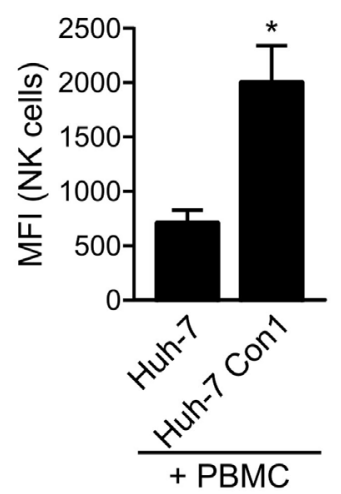

TRAIL
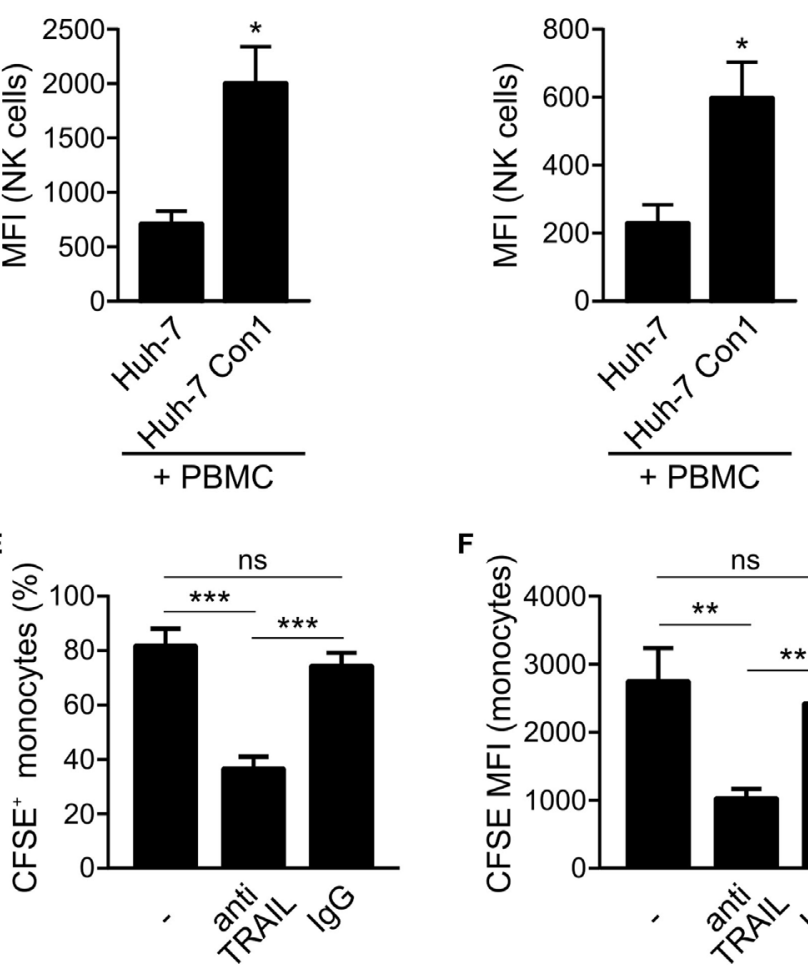

$\mathbf{F}$

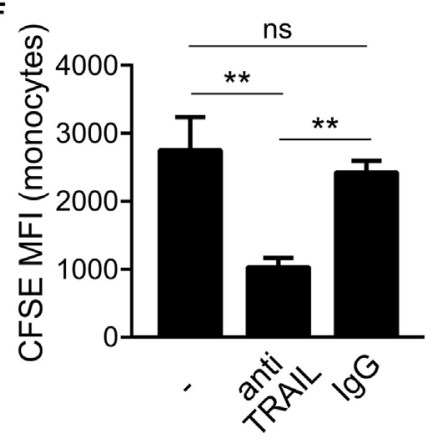

$\overline{\text { Huh-7 Con1 + PBMC }}$

FIGURE 6 | Killing of hepatitis C virus subgenomic replicon cells requires interaction of innate immune cells. (A) Huh-7 Con1 cells were co-cultured with peripheral blood mononuclear cells (PBMCs), purified immune cells [natural killer (NK), plasmacytoid dendritic cells (pDC), or Monocytes (Mono)] or with a recombination of purified NK cells, pDCs and monocytes (NK + pDC + Mono) overnight. Supernatants were collected and measured for lactate dehydrogenase $(\mathrm{LDH})$ activity $(n=3)$. (B) Huh-7 Con1 cells were co-cultured with PBMCs, PBMCs depleted from NK cells (-NK), from pDCs (-pDC), from monocytes (-Mono), or from NK cells and pDCs (-NK -pDC) overnight. Supernatants were collected and measured for LDH activity $(n=4)$. (C) Huh-7 cells were co-cultured with PBMCs overnight. NK cell (CD56+,

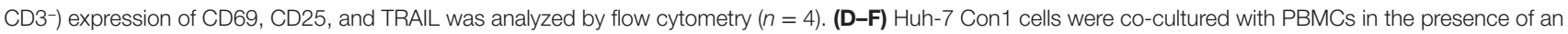
anti-TRAlL antibody or an lgG control antibody (each $10 \mu \mathrm{g} / \mathrm{ml}$ ) overnight. LDH release was measured in the supernatant (D), percentage of CFSE+ monocytes (E), and carboxyfluorescein succinimidyl ester (CFSE) mean fluorescence intensity (MFI) of monocytes (F) was determined $(n=4)$.

CD69 and CD25 in co-culture of Huh-7 Con1 cells with PBMCs. Also, TRAIL expression increased on NK cells in the co-culture setup (Figure 6C). As TRAIL is known to induce apoptosis, we investigated if blocking of TRAIL decreases killing and subsequent uptake of Huh-7 Con 1 cells. Indeed, when blocking TRAIL with a specific antibody, killing was reduced from 40 to $6 \%$ (Figure 6D). Similarly, the number of CFSE positive monocytes and the CFSE MFI of monocytes were significantly lower (Figures 6E,F).

\section{Activated PBMCs Only Target HCV SGR Cells, but Not Naïve Huh-7 Cells}

Since TRAIL expression on NK cells leads to killing of HCV SGR cells, we next determined TRAIL receptor expression in 
Huh-7 and in Huh-7 Con1 cells. However, no differences in expression were observed for the pro-apoptotic receptors DR4 and DR5 and the anti-apoptotic receptor DcR2 at mRNA levels (Figures S4A-C in Supplementary Material), which was also confirmed by flow cytometry analysis (data not shown). DcR1 was not expressed. As no overt difference in expression of receptors for TRAIL could be observed between Huh-7 and Huh-7 Con 1 cells, we wondered whether activated PBMCs would retain their specificity for $\mathrm{HCV}$-infected cells or might then unspecifically kill Huh-7 cells. Therefore, a triple co-culture of Huh-7, Huh-7 Con1 cells and PBMCs was established. In order to verify that the PBMCs were activated in this setup, we analyzed IFN $\alpha$ and IFN $\gamma$, which expectedly were induced. Moreover, NK cell activation markers CD25 and CD69 were also upregulated (Figures S5A-D in Supplementary Material).
Secretion of IFNs and expression of CD25 and CD69 was lower compared to the co-culture of PBMCs and Huh-7 Con 1 cells, as in the triple co-culture only half the amount of Huh-7 Con 1 cells was present. Then we analyzed whether Huh-7 cells would be killed in this triple co-culture setup in which PBMCs were activated by the presence of Huh-7 Con 1 cells. Huh-7 cells were stained with CytoRed and Huh-7 Con 1 cells were stained with CFSE. After overnight co-culture with PBMCs, monocytes were analyzed for CFSE and CytoRed positivity. Strikingly, monocytes turned CFSE positive indicating uptake of Huh-7 Con1 remnants, yet CytoRed staining was only at background levels (Figures 7A-C). Thus, even in a triple co-culture, killing and uptake remained specific for HCV SGR cells. To exclude any effects of the dyes, staining of Huh-7 and Huh-7 Con 1 cells was also performed vice versa with similar results (Figures S5E-G

A

Huh-7 + Huh-7 Con1 + PBMC

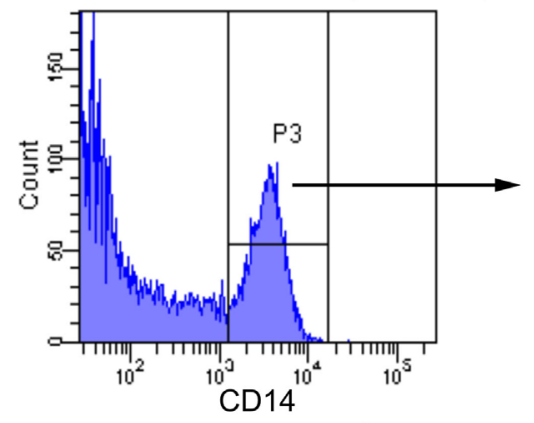

B

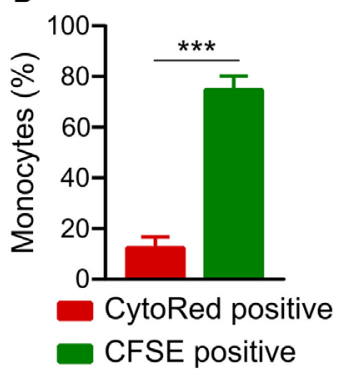

C

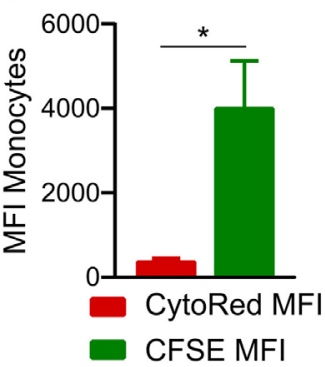

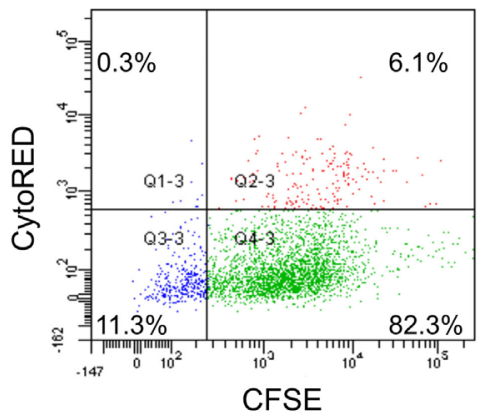

D

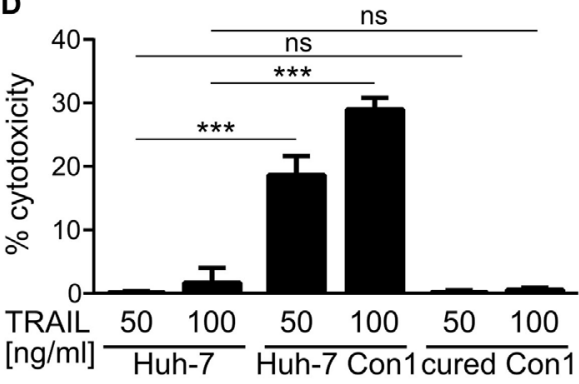

E

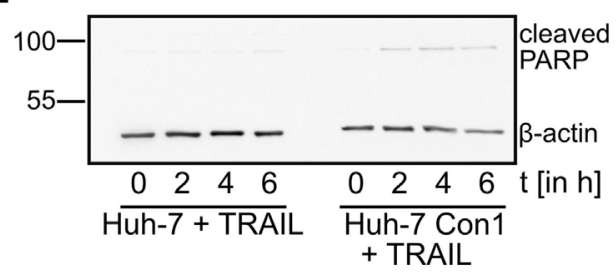

$\mathbf{F}$

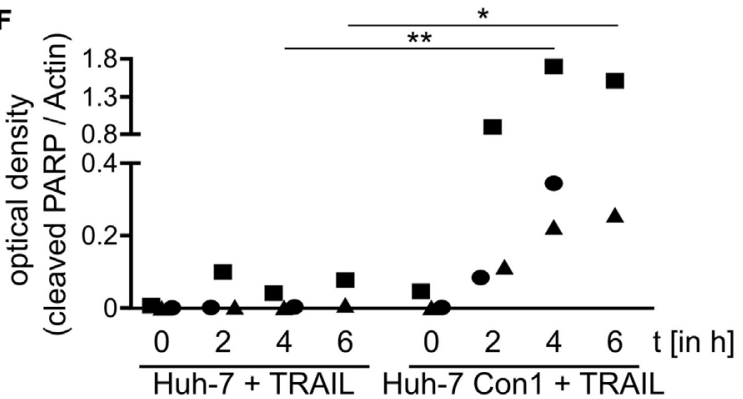

FIGURE 7 | Hepatitis C virus (HCV) specific killing by activated peripheral blood mononuclear cells (PBMCs). (A-C) CytoRED stained Huh-7 cells were co-cultured with carboxyfluorescein succinimidyl ester (CFSE) stained Huh-7 Con1 cells and PBMCs overnight. The percentage of CytoRED and CFSE positive monocytes (B) as well as the CytoRED and CFSE mean fluorescence intensity (MFI) (C) of monocytes were measured $(n=3)$. (D) Huh-7 cells were incubated with recombinant TRAIL at the indicated concentrations overnight. Supernatants were measured for lactate dehydrogenase (LDH) activity $(n=3)$. (E,F) Huh-7 and cells were incubated with recombinant TRAIL (100 ng/ml) for the indicated time points. Poly (ADP-ribose) polymerase (PARP) cleavage was analyzed by Western Blot $(n=2-3)$. 
in Supplementary Material). The results indicate that PBMCs specifically kill HCV SGR cells and despite of their activation by HCV SGR cells they do not target Huh-7 control cells present in the same culture.

\section{HCV SGR Cells Are Sensitive to TRAIL-Induced Apoptosis}

Although Huh-7 and Huh-7 Con1 cells displayed similar TRAIL receptor expression (Figures S4A-C in Supplementary Material), PBMCs specifically killed Huh-7 Con1 cells in a TRAIL dependent manner (Figure 6D). Hence, we hypothesized that HCV SGR cells might be more sensitive to TRAIL induced apoptosis. To test this, recombinant TRAIL was added to Huh7, Huh-7 Con 1 and cured Con 1 cells. While Huh-7 and cured Con 1 cells were not killed by TRAIL treatment, Huh-7 Con 1 cells were killed (Figure 7D). Noteworthy, addition of $\mathrm{TNF} \alpha$ neither induced apoptosis in Huh-7 nor in Huh-7 Con 1 cells (Figure S6 in Supplementary Material). To confirm the finding of increased TRAIL sensitivity in Huh-7 Con 1 cells by an independent assay, cleavage of PARP was examined after addition of recombinant TRAIL. As seen in the PBMC co-culture, cleaved PARP was only detected in Huh-7 Con 1 cells (Figures 7E,F). Thus, TRAIL sensitization in HCV SGR cells explains specific killing by PBMCs.

\section{DISCUSSION}

The results of this study highlight the importance of the interplay of different innate immune cells to initiate an efficient, rapid and specific response against HCV-infected cells. The rapid response within the short time frame of an overnight co-culture points out that the anti-viral response is elicited by innate and not by adaptive immunity. Of course adaptive immunity plays an important role for the defense of HCV infection, yet in this study, we focused on the less well explored role of early innate immune responses. Complete PBMCs within $24 \mathrm{~h}$ were able to induce apoptosis and clear HCV SGR cells (Figures 2 and 5). In contrast, killing activity of purified subsets of immune cells against HCV SGR cells was substantially lower (Figure 6A). Accordingly, the uptake of Huh-7 Con 1 cells by purified monocytes was weaker compared to complete PBMCs. However, activity of purified monocytes against Huh-7 Con 1 cells still was higher compared to Huh-7 control cells (Figure 4A), indicating that monocytes play an active role in the anti-viral response. Nevertheless, presence and activation of pDCs and NK cells remained crucial for the most efficient rapid killing via TRAIL expression by NK cells (Figure 6), arguing for the need of a precisely controlled interplay.

We observed that an efficient anti-viral response against $\mathrm{HCV}$ relied on dual roles of activated immune cells. First, they directly target $\mathrm{HCV}$-infected cells, e.g., by secretion of IFNs (Figures 1A,B) or by upregulation of TRAIL on NK cells (Figure 6). Second, immune cells interact with each other and thereby increase innate activity and specificity. Of note, the observed effects did only occur with HCV SGR but not with DV and HAV SGR cells. The latter two were not killed by PBMCs (Figure 3D). The lack of PBMC activation by DV and HAV
SGR cells can be explained by reduced secretion of viral RNA compared to HCV SGR cells.

Natural killer cells cannot be activated directly by HCV SGR cells: it was reported that in the co-culture of HCV SGR cells with PBMCs, NK cells can be activated by IL-18 derived from monocytes (7). Another study confirmed the role of monocytes in NK activation, but observed that $\mathrm{pDC}$ derived IFN $\alpha$ was more important to activate NK cells (8). In this study, purified NK cells did not kill Huh-7 Con1 cells (Figure 6A) and did not secrete IFN $\gamma$ in co-culture (data not shown). But our results show that IFN $\gamma$ from NK cells increased the uptake of Huh-7 Con 1 cells by purified monocytes (Figures 4E,F), proving that NK cells in turn also influence the response of monocytes.

IFN $\alpha$ from pDCs boosted the uptake of HCV SGR cell remnants by purified monocytes even more (Figures $4 \mathrm{E}, \mathrm{F}$ ), indicating that monocyte action is enhanced by pDC activation. Moreover, published data show that TRAIL expression of NK cells is dependent on $\operatorname{IFN} \alpha(23,24)$. It was shown that purified pDCs secrete IFN $\alpha$ in co-culture with $\mathrm{HCV}$-infected cells by recognition of HCV RNA via TLR7 (6). We could reproduce activation of purified pDCs in our study (data not shown) and confirmed dependency on endosomal TLRs (Figure 1C). However, pDC activation alone was not sufficient to kill HCV SGR cells, interaction with NK cells and monocytes was additionally necessary (Figure 6A). Thus, IFN $\alpha$ is not sufficient to kill HCV replicating cells, yet contributes by activating additional innate immune players (Figures 4E,F).

Monocytes can also sense HCV-infected cells and respond by inflammasome activation (7). Furthermore, monocyte-derived macrophages were shown to recognize HCV dsRNA via TLR3 (25). In line with this, we observed that purified monocytes were able to take up remnants of Huh-7 Con 1 cells (Figure 4A), arguing for a direct recognition of HCV replicating cells by monocytes. The interactions between HCV SGR cells and different innate immune cells are summarized in Figure 8.

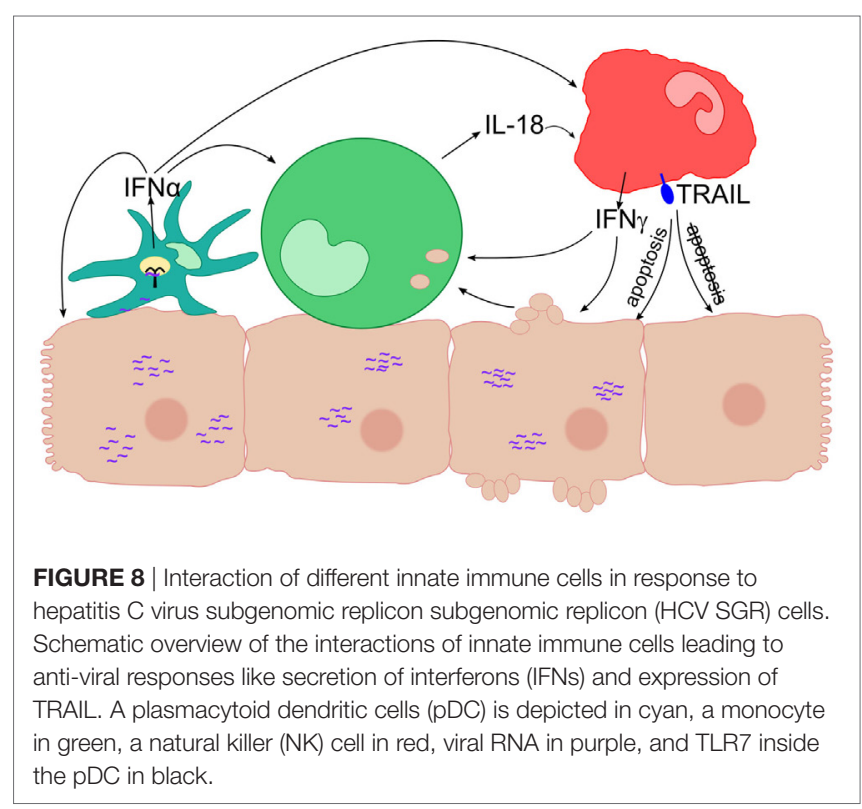


Regarding the activation of immune cells by HCV SGR cells, we show that cell-cell contacts are required, since mechanical agitation significantly reduced IFN $\alpha$ secretion (Figure 1E) and no IFN $\alpha$ and IFN $\gamma$ was produced when PBMCs were separated from HCV SGR cells in a transwell (data not shown). Thus, we confirm previous findings on the importance of cell-cell contacts $(6,21)$. Dreux et al. showed that also exosomes released from $\mathrm{HCV}$-infected cells can activate purified pDCs; mechanistically, activation of pDCs occured due to transfer of viral RNA (22). However, we did not observe stimulation of complete PBMCs by exosomes (data not shown), which highlights the necessity for cell-cell contacts. We speculate that not only close contacts between immune cells and HCV SGR cells are important but also close interactions between immune cells are necessary for their mutual interaction discussed before. In case of full activation of PBMCs, apoptosis was induced in Huh-7 Con 1 cells as shown by cleaved PARP assays and caspase inhibition experiments (Figure 5). Mechanistically we found an upregulation of TRAIL on NK cells in co-culture of PBMCs with Huh-7 Con 1 cells (Figure 6C). Although blocking TRAIL significantly reduced killing and uptake of Huh 7 Con 1 cells (Figures 6D-F), neither kill nor uptake were completely lost. This suggests that also other mechanisms contribute to killing of HCV SGR cells.

The expression of TRAIL on NK cells in the HCV context has been discussed controversially, since TRAIL on the one hand can kill infected cells but also targets liver explants from diseased patients due to upregulation of TRAIL receptors DR4 and DR5 (26). In contrast, in a different study, no upregulation of DR4 and DR5 was found on hepatocytes in vivo during chronic hepatitis (27). Here, we analyzed TRAIL receptor expression in Huh-7 and in Huh-7 Con 1 cells and found no difference in pro-apoptotic TRAIL receptors DR4 and DR5 and in the anti-apoptotic DcR2 receptor (Figure S4 in Supplementary Material). Despite similar TRAIL receptor expression, naïve Huh-7 cells were not targeted by PBMCs that were activated by Huh-7 Con 1 cells in a triple coculture (Figures 7A-C), indicating a specific kill of infected cells. This was confirmed by recombinant TRAIL addition to Huh-7 cells, where only Huh-7 Con 1 cells were driven into apoptosis (Figures 7D,E). According to a previous study, TRAIL sensitization might arise from mitochondrial damage in HCV-infected cells, inducing a cell intrinsic apoptosis pathway. Together with the extrinsic apoptotic signaling via TRAIL, the infected cell is finally driven into apoptosis (28).

Next to the TRAIL sensitization of HCV replicating cells, we speculate that also close interactions between innate immune cells contribute to specific killing of infected cells. We hypothesize that NK cell activation occurs at sites where pDCs and monocytes are activated by sensing HCV-infected cells. Thereby, the interaction of different cells helps to increase specificity by coupling specific innate recognition means (e.g., pDC activation via TLR7 or monocyte activation via TLR3) to the more unspecific effector means (e.g., TRAIL expression by NK cells).

The necessity of precisely controlled spatial and temporal interactions of multiple innate immune cells might give rise to the speculation that full innate immune activation is difficult to achieve. This could contribute to lack of clearance and chronicity of infection in $70-80 \%$ of $\mathrm{HCV}$-infected patients. While in our in vitro model, direct interactions between different innate immune cells and with multiple HCV-replicating cells are possible, in vivo a longer period of time might be required for activation and interaction of multiple innate immune cells. It could be speculated that in HCV-infected patients that spontaneously clear the virus (around 25\%), combined activation of innate immune cells might be established before the virus is able to exert its immune-inhibitory functions described for several immune cells (29).

In summary, this study demonstrates how innate immune cells interact with each other in response to HCV-infected cells. Upon recognition of viral RNA that triggers pDC and monocyte activation, this interaction enables further mutual activation of immune cells. Thereby, an efficient response is initiated that eventually leads to killing of HCV-infected cells via TRAIL expression on NK cells and other yet unknown mechanisms. Importantly, we show that innate immune cells activated by HCV do not target uninfected cells. Whether similar interactions between innate immune cells also occur in the infected liver is subject of further analysis. Although not addressed in this study, the combined activation of innate immune cells might also play an important role for the onset and magnitude of adaptive immunity. A rapid and concerted action of innate and adaptive immunity might explain why some patients spontaneously clear HCV before chronic infection is established in which HCV inhibits several immune responses.

\section{ETHICS STATEMENT}

This study was carried out in accordance with the recommendations of the local Ethic Committee Heidelberg. All subjects gave written informed consent in accordance with the Declaration of Helsinki. The protocol was approved by the local Ethic Committee Heidelberg.

\section{AUTHOR CONTRIBUTIONS}

$\mathrm{AD}$ and VL developed the concept and design of this study. VK, OG, and TE designed experiments. VK, OG, SE, FL, and GW performed experiments and analysis. VK wrote the article with support from $\mathrm{AD}$ and VL.

\section{FUNDING}

This work was supported by a grant from the Excellence Cluster "Cellular Networks" of University Heidelberg.

\section{SUPPLEMENTARY MATERIAL}

The Supplementary Material for this article can be found online at http://journal.frontiersin.org/article/10.3389/fimmu.2017.01238/ full\#supplementary-material. 


\section{REFERENCES}

1. Hajarizadeh B, Grebely J, Dore GJ. Epidemiology and natural history of HCV infection. Nat Rev Gastroenterol Hepatol (2013) 10:553-62. doi:10.1038/ nrgastro.2013.107

2. Lau DT-Y, Fish PM, Sinha M, Owen DM, Lemon SM, Gale M. Interferon regulatory factor-3 activation, hepatic interferon-stimulated gene expression, and immune cell infiltration in hepatitis C virus patients. Hepatology (2008) 47:799-809. doi:10.1002/hep.22076

3. Norris S, Collins C, Doherty DG, Smith F, McEntee G, Traynor O, et al. Resident human hepatitis lymphocytes are phenotypically different from circulating lymphocytes. J Hepatol (1998) 28:84-90. doi:10.1016/S0168-8278(98) 80206-7

4. Vollmar B, Menger MD. The hepatic microcirculation: mechanistic contributions and therapeutic targets in liver injury and repair. Physiol Rev (2009) 89:1269-339. doi:10.1152/physrev.00027.2008

5. Kita H, Van De Water J, Gershwin ME, Mackay IR. The lymphoid liver: considerations on pathways to autoimmune injury. Gastroenterology (2001) 120:1485-501. doi:10.1053/gast.2001.22441

6. Takahashi K, Asabe S, Wieland S, Garaigorta U, Gastaminza P, Isogawa M, et al. Plasmacytoid dendritic cells sense hepatitis C virus-infected cells, produce interferon, and inhibit infection. Proc Natl Acad Sci U S A (2010) 107:7431-6. doi:10.1073/pnas.1002301107

7. Serti E, Werner JM, Chattergoon M, Cox AL, Lohmann V, Rehermann B. Monocytes activate natural killer cells via inflammasome-induced interleukin 18 in response to hepatitis C virus replication. Gastroenterology (2014) 147:209-20.e3. doi:10.1053/j.gastro.2014.03.046

8. Zhang S, Saha B, Kodys K, Szabo G. IFN- $\gamma$ production by human natural killer cells in response to $\mathrm{HCV}$-infected hepatoma cells is dependent on accessory cells. J Hepatol (2013) 59:442-9. doi:10.1016/j.jhep.2013.04.022

9. Frese M, Pietschmann T, Moradpour D, Haller O, Bartenschlager R. Interferon-alpha inhibits hepatitis $\mathrm{C}$ virus subgenomic RNA replication by an MxA-independent pathway. J Gen Virol (2001) 82:723-33. doi:10.1099/ 0022-1317-82-4-723

10. Frese M, Schwärzle V, Barth K, Krieger N, Lohmann V, Mihm S, et al. Interferon-gamma inhibits replication of subgenomic and genomic hepatitis C virus RNAs. Hepatology (2002) 35:694-703. doi:10.1053/jhep.2002. 31770

11. Metz P, Dazert E, Ruggieri A, Mazur J, Kaderali L, Kaul A, et al. Identification of type I and type II interferon-induced effectors controlling hepatitis C virus replication. Hepatology (2012) 56:2082-93. doi:10.1002/hep. 25908

12. Rehermann B, Nascimbeni M. Immunology of hepatitis B virus and hepatitis C virus infection. Nat Rev Immunol (2005) 5:215-29. doi:10.1038/ nri1573

13. Lohmann V, Körner F, Koch J-O, Herian U, Theilmann L, Bartenschlager R. Replication of subgenomic hepatitis C virus RNAs in a hepatoma cell line. Science (1999) 285:110-3. doi:10.1126/science.285.5424.110

14. Lohmann V, Hoffmann S, Herian U, Penin F, Bartenschlager R. Viral and cellular determinants of hepatitis $\mathrm{C}$ virus RNA replication in cell culture. J Virol (2003) 77:3007-19. doi:10.1128/JVI.77.5.3007-3019.2003

15. Grünvogel O, Esser-Nobis K, Reustle A, Schult P, Müller B, Metz P, et al. DDX60L is an interferon-stimulated gene product restricting hepatitis $\mathrm{C}$ virus replication in cell culture. J Virol (2015) 89:10548-68. doi:10.1128/ JVI.01297-15

16. Kumar A, Buhler S, Selisko B, Davidson A, Mulder K, Canard B, et al. Nuclear localization of dengue virus nonstructural protein 5 does not strictly correlate with efficient viral RNA replication and inhibition of type I interferon signaling. J Virol (2013) 87:4545-57. doi:10.1128/JVI. 03083-12
17. Esser-Nobis K, Harak C, Schult P, Kusov Y, Lohmann V. Novel perspectives for hepatitis A virus therapy revealed by comparative analysis of hepatitis $\mathrm{C}$ virus and hepatitis A virus RNA replication. Hepatology (2015) 62:397-408. doi:10.1002/hep. 27847

18. Blight KJ, McKeating JA, Rice CM. Highly permissive cell lines for subgenomic and genomic hepatitis C virus RNA replication. J Virol (2002) 76:13001-14. doi:10.1128/JVI.76.24.13001-13014.2002

19. Pietschmann T, Kaul A, Koutsoudakis G, Shavinskaya A, Kallis S, Steinmann E, et al. Construction and characterization of infectious intragenotypic and intergenotypic hepatitis C virus chimeras. Proc Natl Acad Sci U S A (2006) 103:7408-13. doi:10.1073/pnas.0504877103

20. Balta E, Stopp J, Castelletti L, Kirchgessner H, Samstag Y, Wabnitz GH. Qualitative and quantitative analysis of PMN/T-cell interactions by InFlow and super-resolution microscopy. Methods (2017) 112:25-38. doi:10.1016/j. ymeth.2016.09.013

21. Zhang S, Kodys K, Babcock GJ, Szabo G. CD81/CD9 tetraspanins aid plasmacytoid dendritic cells in recognition of hepatitis $C$ virus-infected cells and induction of interferon-alpha. Hepatology (2013) 58:940-9. doi:10.1002/ hep. 25827

22. Dreux M, Garaigorta U, Boyd B, Décembre E, Chung J, Whitten-Bauer C, et al. Short-range exosomal transfer of viral RNA from infected cells to plasmacytoid dendritic cells triggers innate immunity. Cell Host Microbe (2012) 12:558-70. doi:10.1016/j.chom.2012.08.010

23. Ahlenstiel G, Titerence RH, Koh C, Edlich B, Feld JJ, Rotman Y, et al. Natural killer cells are polarized toward cytotoxicity in chronic hepatitis $\mathrm{C}$ in an interferon- $\alpha$-dependent manner. Gastroenterology (2010) 138:325-35.e2. doi:10.1053/j.gastro.2009.08.066

24. Stegmann KA, Björkström NK, Veber H, Ciesek S, Riese P, Wiegand J, et al. Interferon- $\alpha$-induced TRAIL on natural killer cells is associated with control of hepatitis C virus infection. Gastroenterology (2010) 138:1885-97.e10. doi:10.1053/j.gastro.2010.01.051

25. Zhou Y, Wang X, Sun L, Zhou L, Ma T-C, Song L, et al. Toll-like receptor 3-activated macrophages confer anti-HCV activity to hepatocytes through exosomes. FASEB J (2016) 30(12):4132-40. doi:10.1096/fj.201600696R

26. Volkmann X, Fischer U, Bahr MJ, Ott M, Lehner F, MacFarlane M, et al. Increased hepatotoxicity of tumor necrosis factor-related apoptosis-inducing ligand in diseased human liver. Hepatology (2007) 46:1498-508. doi:10.1002/ hep. 21846

27. Mundt B, Kühnel F, Zender L, Paul Y, Tillmann H, Trautwein C, et al. Involvement of TRAIL and its receptors in viral hepatitis. FASEB J (2003) 17:94-6. doi:10.1096/fj.02-0537fje

28. Lan L, Gorke S, Rau SJ, Zeisel MB, Hildt E, Himmelsbach K, et al. Hepatitis C virus infection sensitizes human hepatocytes to TRAIL-induced apoptosis in a caspase 9-dependent manner. J Immunol (2008) 181:4926-35. doi:10.4049/ jimmunol.181.7.4926

29. Szabo G, Chang S, Dolganiuc A. Altered innate immunity in chronic hepatitis C infection: cause or effect? Hepatology (2007) 46:1279-90. doi:10.1002/ hep. 21938

Conflict of Interest Statement: The authors declare that the research was conducted in the absence of any commercial or financial relationships that could be construed as a potential conflict of interest.

Copyright (c) 2017 Klöss, Grünvogel, Wabnitz, Eigenbrod, Ehrhardt, Lasitschka, Lohmann and Dalpke. This is an open-access article distributed under the terms of the Creative Commons Attribution License (CC BY). The use, distribution or reproduction in other forums is permitted, provided the original author(s) or licensor are credited and that the original publication in this journal is cited, in accordance with accepted academic practice. No use, distribution or reproduction is permitted which does not comply with these terms. 